\title{
Persistent atrial fibrillation is not associated with thrombomodulin level increase in efficiently anticoagulated patients
}

Beata Wożakowska-Kapłon ${ }^{1,2}$, Radoslaw Bartkowiak', Urszula Grabowska ${ }^{3}$, Grażyna Janiszewska ${ }^{4}$

${ }_{1}^{1} 1^{\text {st }}$ Clinical Department of Cardiology, Swietokrzyskie Centre of Cardiology, Kielce, Poland ${ }^{2}$ Faculty of Health Studies, University of Humanities and Science, Kielce, Poland ${ }^{3}$ Central Laboratory, Swietokrzyskie Centre of Cardiology, Kielce, Poland ${ }^{4}$ Chair of Medical Biochemistry, Medical University of Lodz, Poland

Submitted: 10 March 2010

Accepted: 21 April 2010

Arch Med Sci 2010; 6, 6: 887-891

DOI: $10.5114 /$ aoms.2010.19297

Copyright (c) 2010 Termedia \& Banach

\section{Abstract}

Introduction: Atrial fibrillation (AF) is the most common arrhythmia and leads to a five-fold increased risk of stroke compared to persons with sinus rhythm. A soluble form of thrombomodulin (STM) is a recognized marker of endothelial dysfunction and may contribute to the hypercoagulable state in AF. The aim of the study was to evaluate plasma concentration of sTM in persistent AF patients before and after sinus rhythm recovery following direct current cardioversion (CV).

Material and methods: In 45 effectively anticoagulated consecutive patients, with persistent non-valvular AF, and normal left ventricular function, CV was performed. Blood samples for STM assessment were collected twice: 24 hours before and 24 hours after CV.

Results: In 43 patients sinus rhythm was obtained. The mean plasma STM level was significantly lower in AF patients compared to the control group with sinus rhythm and without anticoagulation $(38.5 \pm 9.9 \mathrm{ng} / \mathrm{ml}$ vs. $44.1 \pm 9.1 \mathrm{ng} / \mathrm{ml}$, $p=0.04$ ). Plasma STM levels did not change 24 hours after successful CV $(36.7 \pm 9.5 \mathrm{ng} / \mathrm{ml}$ vs. $38.5 \pm 9.9 \mathrm{ng} / \mathrm{ml}, p=0.16)$.

Conclusions: Plasma STM concentration was lower in patients with persistent $\mathrm{AF}$ and normal left ventricle systolic function than in patients with sinus rhythm, presumably due to chronic oral anticoagulant therapy in the AF group. CV has no impact on STM plasma level evaluated 24 hours after sinus rhythm restoration.

Key words: atrial fibrillation, thrombomodulin, cardioversion

\section{Introduction}

Atrial fibrillation (AF) is the most common arrhythmia, affecting $>5 \%$ of the population $>65$ years of age, with a prevalence that doubles with every decade of life [1]. The most important problem with AF is the risk of stroke. Patients with AF have a five-fold increased risk of stroke compared to those in sinus rhythm [2]. The loss of mechanical atrial contraction leads to altered blood flow characteristics that can result in clot formation, almost exclusively in the left atrial appendage. Recently it has been reported that $\mathrm{AF}$ is accompanied by a hypercoagulable state that may contribute to the development of atrial thrombi and thromboembolism [3-10]. The

\author{
Corresponding author: \\ Beata Wożakowska-Kapłon, \\ MD, PhD \\ $1^{\text {st }}$ Clinical Department \\ of Cardiology \\ Swietokrzyskie Centre \\ of Cardiology \\ Grunwaldzka 45 \\ 25-736 Kielce, Poland \\ Phone/fax +48 413671396 \\ E-mail: \\ bw.kaplon@poczta.onet.pl
}


mechanisms responsible for this hypercoagulable state remain undefined. However, increased thrombogenesis, abnormal haemostasis, fibrinolysis and platelet functions, and atrial and arterial endothelial damage or dysfunction, could contribute to the hypercoagulable state in AF [3-10]. Randomized controlled trials have convincingly demonstrated that the risk of AF-associated thromboembolic events (predominantly strokes) can be reduced by $64 \%$ by therapy with vitamin $\mathrm{K}$ antagonists [11]. Direct current cardioversion (CV) of AF can be associated with a transient mechanical dysfunction of the left atrium ("atrial stunning"). Increased atrial stasis connected with abnormal haemostasis would fulfil components of Virchow's triad leading to the development of atrial thrombus and thromboembolism. Soluble thrombomodulin (sTM), among other endothelial plasma indexes (e.g. von Willebrand factor, nitrate/nitrite product NOx, soluble E-selectin), is considered to be a plasma endothelial damage or dysfunction marker. However, STM is a natural anticoagulant on the membranes of endothelial cells which acts as a receptor for thrombin [12]. Therefore, the TM protein C complex plays a crucial role in maintaining normal blood fluidity and preventing intravascular thrombosis [12]. The aim of the present study was to investigate whether persistent AF in anticoagulated patients creates a prothrombotic state, whether CV itself causes endothelial damage and leads to increase of STM, or if sinus rhythm restoration causes changes in STM levels.

\section{Material and methods}

Direct current CV was performed in 45 consecutive patients with persistent non-valvular $A F$ with normal left ventricular ejection fraction (LVEF) in echocardiographic examinations ( $\geq 50 \%)$. In all patients, left ventricular ejection fraction (LVEF) and left atrial (LA) diameter were assessed by echocardiography examinations before CV. The procedure was unsuccessful in 2 cases. Finally 43 patients ( 35 men and 8 women, aged 59 \pm 11.8 years) were included in the study. Patients in the study group had normal blood pressure, no symptoms of heart failure and they had preserved left ventricular systolic function. The control group comprised 20 patients with sinus rhythm, without a history of AF and not undergoing anticoagulation therapy. They were compatible in age and gender, and they did not differ from the study group in baseline clinical (hypertension, coronary disease or diabetes) and echocardiographic data. All AF patients were scheduled for electrical CV and they received an oral anticoagulant (acenocoumarol) for at least 4 weeks (international normalized ratio, INR, between 2.0 and 3.0) before admission. Pharmacological therapy was continued before and after CV. CV was performed under intravenous general anaesthesia (fentanyl 0.05-0.1 mg, etomidate $0.2 \mathrm{mg} / \mathrm{kg}$ ). CV was performed by delivering $\mathrm{R}$ wave synchronized biphasic shocks with a Medtronic Physio-Control Lifepak 12 external defibrillator. The initial shock energy was $100 \mathrm{~J}$, with a subsequent increment to $200 \mathrm{~J}$ and a further $200 \mathrm{~J}$ in the event of CV failure. Sinus rhythm maintenance was assessed in all patients on the $30^{\text {th }}$ day following CV on the basis of an electrocardiogram and the patients' medical history during the previous month. Blood samples for plasma STM assessment were collected in the morning, from the cubital vein twice: 24 hours before and 24 hours after CV. All specimens were collected in tubes containing EDTA (ethylenediaminetetraacetic acid) $1.5 \mathrm{~g} / \mathrm{l}$. The plasma was separated by centrifugation (at $2500 \mathrm{rpm}$ ) for $20 \mathrm{~min}$ at $4^{\circ} \mathrm{C}$ and stored at $-20^{\circ} \mathrm{C}$ until measurement. Soluble TM was determined using enzyme-linked immunosorbent assay (nanograms per millilitre; Diagnostica Stago). Inter- and intraassay coefficients of variation were $5 \%$ and $7 \%$ respectively.

All values are expressed as mean \pm standard deviation. The paired Student's $t$ test was used to compare values before and after CV. The Pearson correlation coefficient was used to determine the correlation between continuous variables. A $p$ value $<0.05$ was considered statistically significant. All analyses were performed using the Med Calc program, version $4.16 \mathrm{~g}$.

\section{Results}

\section{Study population characteristics}

The clinical characteristics of the study population were typical for patients with persistent AF who are referred for CV. The majority of them were men (81\%) with a history of hypertension (63\%). Symptomatic coronary artery disease was diagnosed in $19 \%$ of patients and $7 \%$ of the study group suffered from diabetes (some of the patients had more than one of the above-mentioned conditions). Patients with lone AF constituted 20\% of the study population. The mean AF duration was $12.3 \pm 15.3$ weeks. Echocardiographic examination of patients included in the study revealed moderate atrial dilatation (43.7 $\pm 5.9 \mathrm{~mm}$ ). Mean LVEF assessed by echocardiography was in the normal range $(57.3 \pm 6.1 \%)$. Plasma NT-proANP level in patients with persistent AF was $4.8 \pm 2.9 \mathrm{nmol} / \mathrm{l}$, and 2.8 $\pm 1.2 \mathrm{nmol} / \mathrm{l}, p=0.004$ in the control group. All patients were effectively anticoagulated (INR 2.0-3.0; mean $2.43 \pm 0.4$ ). The baseline characteristics of the study population are listed in table I. All patients who had successful CV (mean energy delivered was $193 \pm 62 \mathrm{~J}$ ) remained in sinus rhythm 
until hospital discharge. Due to sinus rhythm maintenance on the $30^{\text {th }}$ day following CV, patients were divided into two subgroups: group A comprised 24 patients (56\%) maintaining sinus rhythm during 30 days following CV, and group $B$ consisted of 19 patients (44\%) with recurrent AF. There were no significant differences between the two subgroups in clinical and echocardiographic baseline characteristics, or in antiarrhythmic therapy after CV.

\section{Plasma soluble thrombomodulin levels in persistent AF patients}

The mean baseline plasma sTM level was significantly lower in patients with persistent arrhythmia compared to the control group with sinus rhythm and no history of $\mathrm{AF}, 38.5 \pm 9.9 \mathrm{ng} / \mathrm{ml}$ vs. $44.1 \pm 9.1 \mathrm{ng} / \mathrm{ml}, p=0.04$ (Figure 1). There was no correlation between clinical (age, duration of AF, body mass index, heart rate, blood pressure) and echocardiographic data and plasma STM level. Plasma STM levels did not change in 24 hours after successful CV (36.7 $\pm 9.5 \mathrm{ng} / \mathrm{ml}$ vs. $38.5 \pm 9.9 \mathrm{ng} / \mathrm{ml}$, $p=0.16$ ) (Figure 2). Plasma sTM levels before CV did not differ significantly between the sinus rhythm subgroup $A$ and the $A F$ recurrence subgroup $B$ (39.3 $\pm 10.3 \mathrm{ng} / \mathrm{ml}$ vs. $37.4 \pm 9.3 \mathrm{ng} / \mathrm{ml}, p=0.53)$.

\section{Discussion}

The paper presents the results of a study evaluating sTM concentration in the blood in patients with persistent AF and after the recovery of sinus rhythm. The soluble form of thrombomodulin is one of the best known endothelium damage factors. The research conducted in the group of patients with AF indicates that haemodynamic disorders connected with arrhythmia lead to significant dysfunction of the endothelial cells, both in the heart cavities and in the vessels $[4,8]$. In patients with $A F$, an increased STM concentration might be anticipated.

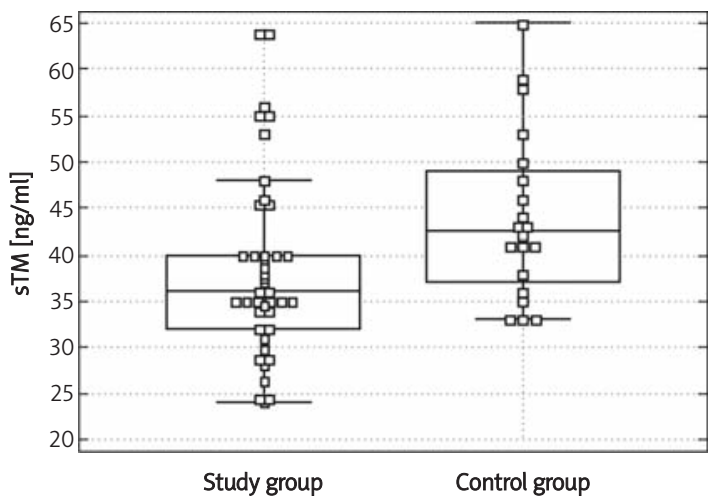

Figure 1. Plasma STM levels in 43 patients with persistent atrial fibrillation and in the control group
Table I. Study group characteristics

\begin{tabular}{|lc|}
\hline $\begin{array}{l}\text { Parameter } \\
\text { Age [years] }\end{array}$ & $\begin{array}{c}\text { Study group, } n=43 \\
\text { X } \pm \text { SD }\end{array}$ \\
\hline Gender, $n$ (\%) & $59.0 \pm 11.6$ \\
\hline women & $8(18.6)$ \\
\hline men & $35(81.4)$ \\
\hline BMI [kg/m²], $n(\%)$ & $5(11.6)$ \\
\hline normal (BMI<25) & $19(44.2)$ \\
\hline verweight (BMI 25-29) & $19(44.2)$ \\
\hline obesity (BMI $\geq 30)$ & $85.4 \pm 13.0$ \\
\hline HR (beats/min) & $130.6 \pm 14.9$ \\
\hline Systolic BP [mmHg] & $80.3 \pm 9.0$ \\
\hline Diastolic BP [mmHg] & $27(62.8)$ \\
\hline Hypertension, $n(\%)$ & $8(18.6)$ \\
\hline Coronary artery disease, $n(\%)$ & $3(7.0)$ \\
\hline Diabetes, $n(\%)$ & $9(20.9)$ \\
\hline Lone AF, $n(\%)$ & $43.7 \pm 5.9 \pm 6.1$ \\
\hline AF duration [weeks] & \\
\hline LVEF [\%] & \\
\hline LA dimension [mm] & \\
\hline
\end{tabular}

$B M I$ - body mass index, $H R$ - heart rate, $B P$ - blood pressure, $A F$ - atrial fibrillation, $L V E F$ - left ventricular ejection fraction, $L A$ - left atrium

In patients with AF who do not use oral anticoagulants, increased STM concentration has been observed in the course of arrhythmia [3, 8]. In the present study the STM concentration in patients with persistent AF was significantly lower than in the patients from the control group. The lower STM concentration in patients with persistent AF is probably the result of long-term use of anticoagulants (vitamin K antagonists), which, apart from the influence they have on plasma coagulation factors, also inhibit the function of thrombocytes

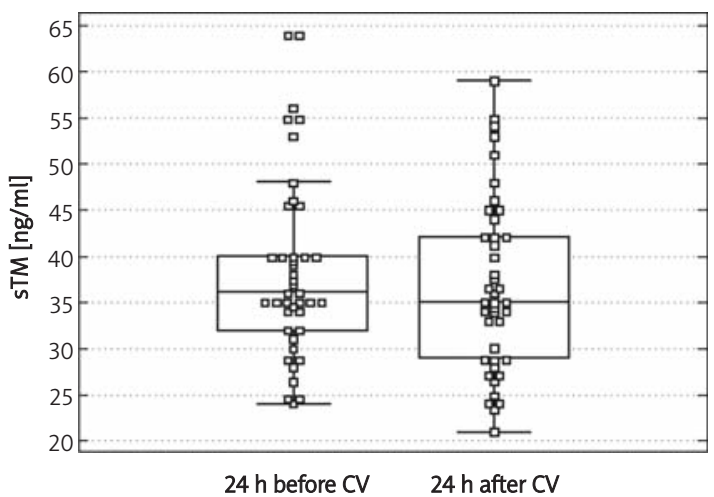

Figure 2. Plasma STM levels before and after successful cardioversion in 43 patients with persistent atrial fibrillation 
and hinder the activity of the endothelium [13]. Ikuma et al. [14] noted that long-term therapy with oral anticoagulants leads to lower STM concentrations in the blood.

Li-Saw-Hee et al. [9] discovered that STM plasma concentration in patients with persistent AF who do not use oral anticoagulants (vitamin $\mathrm{K}$ antagonists) is significantly higher than in control group patients with sinus rhythm. The same authors stated, on the basis of a study of patients with persistent AF with concomitant mitral stenosis (with long-term use of warfarin - INR on average 1.7), that the STM concentration was significantly lower in comparison to the control group of healthy volunteers with normal sinus rhythm [15]. Freestone et al. [16], examining markers of endothelial damage (von Willebrand factor and STM) before and after CV, did not observe increased STM plasma concentrations in persistent AF before CV. At the same time, Marin et al. [3] discovered that STM concentrations in patients with both paroxysmal and persistent AF are significantly higher than in patients with stable coronary disease with sinus rhythm. Attention should be paid to the fact that in that study neither group of patients had used oral anticoagulants for a significant amount of time, and they were administered antiplatelet drugs only.

Finally, Freestone et al. [17] studied 145 patients with AF (paroxysmal, persistent and permanent) and 35 patients with lone AF. Plasma levels of sTM measured by ELISA tests were compared to 40 healthy control subjects in sinus rhythm. Plasma levels of STM in patients with AF were not significantly different (control group: $43 \mathrm{ng} / \mathrm{ml}$ vs. AF group: $31 \mathrm{ng} / \mathrm{ml}, p=0.119)$. There were no differences among the clinical subgroups of patients with paroxysmal, persistent and permanent AF. Patients with lone AF had significantly lower STM concentration compared to the control group. Plasma STM level did not differ significantly among patients with AF after 4 weeks of warfarin treatment.

In our study plasma STM concentration was lower in patients with persistent AF than in control patients with sinus rhythm. The slightly lower STM levels in AF patients may be associated with the presence of an extrinsic anticoagulant (i.e. a vitamin $\mathrm{K}$ antagonist which could alter the expression of this physiological anticoagulant by reducing thrombin formation) or could alternatively be due to the downregulation of thrombomodulin as a result of early endothelial dysfunction. Takeshi et al. [18] revealed that rapid atrial pacing significantly reduced gene expression of TM in the left atrium. Immunohistochemical analysis showed that TM was expressed predominantly in the normal atrial endocardium, presumably preventing local blood coagulation. Rapid atrial pacing downregulated the gene expression in the endocardium, leading to deficiency in anticoagulant barriers. In the examined group of patients with persistent AF there was no association between the basal STM plasma concentration and the age of patients, duration of $A F$, body mass index, heart rate, blood pressure and echocardiographic data. In contrast, Mondillo et al. [4] in a group of patients with lone AF, who did not undergo anticoagulation, reported slightly higher values of sTM than in the controls, and observed a positive linear correlation between left atrial volume and markers of endothelial dysfunction (STM and von Willebrand factor).

Improved function of the endothelium within the heart cavities could be associated with the stabilization of the heart rhythm and improved mechanical heart function after CV. As the recovery of haemodynamic atrial function does not happen directly after $C V$, it can be assumed that the main factor influencing STM concentrations after arrhythmia regression is the stabilization of heart rhythm. On the other hand, as CV is connected with analgesia and the use of electric current, the effect of the procedure itself on the endothelial dysfunction directly after the recovery of sinus rhythm cannot be excluded. The damage of the endothelium may take place during $\mathrm{CV}$, which could intensify the hypercoagulability state. Although changes in plasma sTM levels might be expected, no statistically significant changes after the recovery of sinus rhythm, in the first 24 hours after $\mathrm{CV}$, were observed in our study. Our findings are in accordance with the study of Freestone et al. [16]. They concluded that concentrations of plasma STM and other endothelial markers (von Willebrand factor, soluble E-selectin, nitrate/nitrite products) did not significantly increase after successful CV. All patients were therapeutically anticoagulated in our study and in that of Freestone et al. [16]. Analysing the endothelial damage markers before and after CV, the authors stated that the expression of endothelial dysfunction can be attenuated by the effect of long-term acenocoumarol use. This is a further reason for the necessity to resort to effective anticoagulation in AF patients during a period of at least 4 weeks after the recovery of sinus rhythm.

Persistent atrial fibrillation in efficiently anticoagulated patients is not associated with higher thrombomodulin levels, in comparison to the control group. Successful CV does not lead to significant changes in thrombomodulin concentration in a period of 24 hours after CV.

\section{Acknowledgments}

This study was supported by the Polish Committee for Scientific Research, grant KBN 2 P05B 03426. 


\section{References}

1. Go AS, Hylek EM, Phillips KA, et al. Prevalence of diagnosed atrial fibrillation in adults: national implications for rhythm management and stroke prevention: the AnTicoagulation and Risk Factors in Atrial Fibrillation (ATRIA) Study. JAMA 2001; 285: 2370-5.

2. Wolf PA, Abbott RD, Kannel WB. Atrial fibrillation as an independent risk factor for stroke: the Framingham Study. Stroke 1991; 22: 983-8.

3. Marin F, Roldán V, Climent VE, et al. Plasma von Willebrand factor, soluble thrombomodulin, and fibrin D-dimer concentrations in acute onset non-rheumatic atrial fibrillation. Heart 2004; 90: 1162-6.

4. Mondillo S, Sabatini L, Agricola E, et al. Correlation between left atrial size, prothrombotic state and markers of endothelial dysfunction in patients with lone chronic nonrheumatic atrial fibrillation. Int J Cardiol 2000; 75: 227-32.

5. Kumagai K, Fukunami M, Ohmori M, Kitabatake A, Kamada T, Hoki N. Increased intracardiovascular clotting in patients with chronic atrial fibrillation. J Am Coll Cardiol 1990; 16: 377-80.

6. Lip GY, Lane D, Van Walraven C, Hart RG. Additive role of plasma von Willebrand factor levels to clinical factors for risk stratification of patients with atrial fibrillation. Stroke 2006; 37: 2294-300.

7. Conway DS, Pearce LA, Chin BS, Hart RG, Lip GY. Prognostic value of plasma von Willebrand factor and soluble P-selectin as indices of endothelial damage and platelet activation in 994 patients with nonvalvular atrial fibrillation. Circulation 2003; 107: 3141-5.

8. Takahashi N, Ishibashi Y, Shimada T, et al. Atrial fibrillation impairs endothelial function of forearm vessels in humans. J Card Fail. 2001; 7: 45-54.

9. Li-Saw-Hee FL, Blann AD, Lip GY. A cross-sectional and diurnal study of thrombogenesis among patients with chronic atrial fibrillation. J Am Coll Cardiol 2000; 35: 1926-31.

10. Hammwöhner M, Ittenson A, Dierkes J, et al. Platelet expression of CD40/CD40 ligand and its relation to inflammatory markers and adhesion molecules in patients with atrial fibrillation. Exp Biol Med 2007; 232: 581-9.

11. Hart RG, Pearce LA, Aguilar MI. Meta-analysis: antithrombotic therapy to prevent stroke in patients who have nonvalvular atrial fibrillation. Ann Intern Med 2007; 146: 857-67.

12. Salem HH, Maruyama I, Ishii H, Majerus PW. Isolation and characterization of thrombomodulin from human placenta. J Biol Chem 1984; 259: 12246-51.

13. Roldán V, Marín F, Blann AD, et al. Interleukin-6, endothelial activation and thrombogenesis in chronic atrial fibrillation. Eur Heart J 2003; 24: 1373-80.

14. Ikuma $\mathrm{H}$, Wada $\mathrm{H}$, Mori $\mathrm{Y}$, et al. Hemostatic markers in Japanese patients undergoing anticoagulant therapy under thrombo-test monitoring. Blood Coagul Fibrinolysis 1999; 10: 429-34.

15. Li-Saw-Hee FL, Blann AD, Goldsmith I, Lip GY. Indexes of hypercoagulability measured in peripheral blood reflect levels in intracardiac blood in patients with atrial fibrillation secondary to mitral stenosis. Am J Cardiol 1999; 83: 1206-9.

16. Freestone B, Chong AY, Blann AD, Lip GY. The effects of direct current cardioversion for persistent atrial fibrillation on indices of endothelial damage/dysfunction. Thromb Res 2006; 118: 479-85.

17. Freestone B, Chong AY, Nuttall S, Blann AD, Lip GY. Soluble E-selectin, von Willebrand factor, soluble Thrombomodulin, and total body nitrate/nitrite product as indices of endothelial damage/dysfunction in paroxysmal, persistent, and permanent atrial fibrillation. Chest 2007; 132: 1253-8.

18. Yamashita T, Sekiguchi A, Iwasaki YK, et al. Thrombomodulin and Tissue Factor Pathway Inhibitor in endocardium of rapidly paced rat atria. Circulation 2003; 108: 2450-2. 